\title{
Bypassing Primary Health Care Facilities for Common Childhood Illnesses in Sharg-Alneel Locality in Khartoum State, Sudan 2015
}

\author{
Malaz Elbashir Ahmed ${ }^{1, *}$, Talal Elfadil Mahdi², Nada Jaffar Osman Ahmed ${ }^{3}$ \\ ${ }^{1}$ Community Medicine, Federal Ministry of Health, Directorate General of Human Resources for Health Development, HRH Policy and \\ Planning Director, Khartoum, Sudan \\ ${ }^{2}$ Community Medicine, Director General of the National Health Insurance Fund, Khartoum, Sudan \\ ${ }^{3}$ Community Medicine, Federal Ministry of Health, Directorate General of Primary Health Care, Maternal and Child Health Director, \\ Khartoum, Sudan
}

\section{Email address:}

malazbashirahmed@hotmail.com (M. E.Ahmed), talalelfadil@yahoo.com (T. E. Mahdi), nadajafar@gmail.com (N. J. O. Ahmed)

${ }^{*}$ Corresponding author

\section{To cite this article:}

Malaz Elbashir Ahmed, Talal Elfadil Mahdi, Nada Jaffar Osman Ahmed. Bypassing Primary Health Care Facilities for Common Childhood Illnesses in Sharg- Alneel Locality in Khartoum State, Sudan 2015. Science Journal of Public Health. Vol. 5, No. 2, 2017 , pp. $77-87$. doi: $10.11648 /$ j.sjph.20170502.14

Received: December 17, 2017; Accepted: January 9, 2017; Published: February 9, 2017

\begin{abstract}
In Sudan despite the current implementation of universal health coverage policy, routine monitoring reports highlight that patients frequently bypass Primary Health Care (PHC) facilities in favor of higher-level hospitals, though hospitals are costly and time consuming. The main objective of this study was to study the extent of bypassing the public PHC facilities and factors associated with the decision of caretakers to bypass such facilities seeking care for their under-five year's children with common illnesses in Sharg-Alneel locality, 2015. The study proposed strategies and interventions to the Sudan government -Federal Ministry of Health (FMoH) - to improve PHC service utilization The study was cross- sectional comparative study, interviewer administered questionnaires and facility assessment checklist was used for data collection. The data was analysed using SPSS. The study interviewed 497 caretakers, 87\% of them pursued health care for their children directly from secondary hospitals. The main reasons for bypassing the closest public health facilities were unavailability of doctors, lack of health insurance services and higher cost of services. The proportion of bypassing a PHC facility for child care is significantly associated with child sex, child age, presenting symptoms of diarrhea, fever, difficult breathing and severe vomiting, caretakers' occupation as well as the economic status. In a resource limited country, health policy to achieve universal health coverage is better to focus on quality of care as well as quantity. Community mobilization and interventions to improve access and utilization of quality PHC services are all recommended. Furthermore, more research on bypassing behaviour is also recommended.
\end{abstract}

Keywords: PHC, Child Health Services Utilization, Accessibility, Caretaker

\section{Introduction}

The provision of PHC services in Sudan is arranged across four levels of services according to the population size. Each level is provided by specific type of facilities that has standardized service profile, staffing and supported by a standardized set of management and supportive components. The four levels of service are: community services, family health units, family health centers (FHC) and local hospitals. The five core services provided through those four levels are: treatment of common illness including Integrated Management of Childhood Illnesses (IMCI), vaccination, antenatal care and family planning, growth monitoring and nutrition education and provision of essential drugs. Other PHC services e.g. management of non-communicable diseases, emergency obstetric care, mental health, dental health, eye health,are provided mainly at the level of local 
hospitals [1].

Child health care services in Sudan include: Expanded Program on Immunization (EPI), IMCI, and Nutrition services as well as Maternal, Newborn and Child health services. The implementation of those services in Sudan is fragmented and of limited coverage; the coverage of IMCI is $22 \%$, of EPI $76 \%$ and only $42 \%$ of the population has access to essential MCH services [2].

Sudan is one of seven countries in the Eastern Mediterranean region which has a very high mortality rates in children under the age of five (83 per 1000) [3]. The major causes of morbidity in the under-five children in Sudan are malaria, diarrheal diseases, acute respiratory infections (ARI), vaccine preventable diseases especially measles and the underlying malnutrition. Most of under-5 deaths (58\%) occur in the post-neonatal period and $42 \%$ occur in the neonatal period [2].

Key interventions to prevent and control all the above mentioned leading causes of morbidity and mortality among children are best to be provided and delivered by PHC packed by strong referral system [4-7]. However, several studies [8-11] have documented poor quality of services in most of these facilities causing majority of the care-seekers to bypass them while seeking child care.

According to Sudan referral system, PHC facilities are supposed to be the first contact for mild childhood illnesses. However, in 2011 a KAP survey $(\mathrm{n}=2223)$ indicated that $19 \%$ of the respondents prefer to utilize hospitals directly [12]. In 2013 Khartoum annual health statistical report showed that PHC coverage is 1:13.000. The report also indicated that the total number of out-patients is about 6.289 .568 , of whom $36 \%$ attending at hospital level, $31 \%$ attending FHC, only $3 \%$ attending $\mathrm{FHU}$ and the rest attending at private facilities. Under-5 children represent 1096526 of the total number of out-patients. Only 423437 out of the 2242306 attended the referral hospitals $(19 \%)$ had been referred [13]. Therefore, over $80 \%$ of out-patients in Khartoum approach secondary level directly after feeling of sickness.

Sudan has a high rate of mortality among children under age of 5 caused by diseases that can be simply prevented and treated at PHC facilities which are made easily accessible to the population [3]. Yet, self-referral (bypassing primary care facilities) for common childhood illnesses is a widespread phenomenon. Bypassing primary care level results in overburden of referral facilities and high cost for the care takers and health care system. So there is a general failure in applying a primary health care approach and implementing referral system in Sudan. This study was aimed to 1) measure the proportion of bypassing public PHC facilities among caretakers seeking care for their under-five children 2) determine consistency (illnesses that is difficult to be treated at the primary care facility) of self-referral with the national IMCI guidelines 3 ) identify factors (caretakers, child, illness factors, institutional) associated with bypassing public PHC services. Since PHC services are supposed to be the first contact for common childhood illnesses, the two words bypassing and self-referral will be used interchangeably in this paper.

\section{Methods}

\subsection{Study Design}

This was a cross sectional comparative study to measure the proportion and clients reasons for bypassing PHC facilities. This was complemented by assessment of all primary health facilities bypassed by the interviewed caretakers to explore facility as well as providers factors related to bypassing PHC services.

\subsection{Study Area}

Un-published routine data is indicating that the referral system is inadequately performing at all the 7 localities in Khartoum state. To choose the appropriate study area for conducting our study we have agreed on specific criteria, accordingly Sharg-Alneel locality was selected because it has high reported cases of childhood illnesses, wide range of PHC facilities and also because of its priority locality for FMOH.

Sharg-Alneel locality health care system has three levels, the highest of which are Alban -Jadeed, Om-Dawaban, AbuDleg and Wad-Abu-Salih hospitals with 462 beds at the rate of $1 / 2143$ population and only 16 pediatrics' beds. It serves an estimated population of 989919 at the rate of 1/197984. There are 36 governmental family health centers (FHC) and 53 non-governmental organizations (NGOs). FHC provides the second level of care, and the first level is provided by 50 governmental FHUs. FHC coverage is estimated to be $1 / 11000$ population, if properly utilized, it has a great potential for further health gains for children under five years. There are also one private hospital and 135 private clinics [13].

\subsection{Study Population}

The target population refers to the population from which the sample population was drawn. For this study, the target population was the total number of caretakers seeking care for their under-five children at the four governmental hospitals in Sharg-Alneel locality.

The participants for this study were: caretakers of sick children between 1 month and 5 years, with a history of fever, cough or diarrhoea seeking care at the outpatient/emergency departments of the 4 referral hospitals. The study population also involved the family health centres, family health units at the locality mentioned by the interviewed caretakers, with the aim to examine, in general, the characteristics of facilities intended to be bypassed.

\subsection{Sample Size}

The equation for calculating sample size was as follows:

$$
\mathrm{n}=\mathrm{z}^{2} \mathrm{P}(1-\mathrm{P}) / \mathrm{e}^{2}
$$

Where: 
$\mathrm{Z}=$ level of confidence (1.96)

$\mathrm{P}=$ baseline level of the indicators (proportion of bypassing $\mathrm{PHC}$ facilities) (0.8)

$\mathrm{e}=$ margin of error $(0.05)$

The calculation for sample size was as follows:

$$
\mathrm{N}=\left(1.96^{2}\right) \times 0.8(1-0.8)(0.05)^{2}=246 \times 2=492
$$

492 was the total final sample size from all of the 4 hospital setting. To compute sample size, the average number of patients seen in the various hospitals at Sharg- Alneel locality was obtained from Statistical records which are under the responsibility of the statistics and information department at the Khartoum state ministry of health. 500 questionnaires were collected and 497 analyzed.

Table 1. Sample size per each hospital.

\begin{tabular}{lll}
\hline Hospital Name & $\begin{array}{l}\text { Patients attendants in the } \\
\text { hospital in the last 6 months }\end{array}$ & $\begin{array}{l}\text { Sample } \\
\text { allocation }\end{array}$ \\
\hline Abo delag & 7616 & 40 \\
Wad abosalih & 5882 & 31 \\
Alban jaded & 48978 & 265 \\
Om dawan ban & 29158 & 161 \\
Total & 91634 & 497 \\
\hline
\end{tabular}

\subsection{SamplingTechnique}

According to statistical records obtained, approximately 91634 patients were seen at the 4 hospitals during the last 6 months. These formed the study population as it was from this sampling frame a sample of patients to participate in the study was obtained. Stratified sampling was used. From each of the 4 hospital, setting sample was taken according to the number of outpatients in each hospital. Probability simple random sampling was used. Study samples were selected randomly from all sick children presenting at the outpatient/emergency room, between 9 am and $2 \mathrm{pm}$, with a history of one or more of the following symptoms: fever, cough, difficulty in breathing/fast breathing and diarrhoea or vomiting. Purposive sampling was used for focus group discussion.

\subsection{Data Collection}

The research team included a principal investigator and 4 data collectors. Contact between the team was sustained with regular meetings and phone calls.

\subsection{Data Collection Techniques}

The proportion of bypassing PHC facilities among study population as well as all variables in the questionnaire such as demographic characteristics and reasons behind bypassing PHC were explored using the following data collection techniques:

\subsubsection{Interviewing}

The interviewers were personally managing the study at outpatient/emergency room. The purpose of the study was clarified and the participant was asked for her/his consent to take part in the study. Before progressing with the interview, consent form was read to and signed by the participant. The interviewwas carried out using questionnaire with fixed list of questions.

\subsubsection{Observing}

Public family health centers and units mentioned by the bypassed study participants within Sharg-Alneel locality were selected for the assessment. Interviewed caretakers mentioned names of 32 primary public health facilities close to their homes and which could have been used by them. In preparing for the assessment tool, principal investigator reviewed the National IMCI program tools developed for health worker follow up after training and assessment during supervision. Then the principal investigator drafted an observation and facility inventory checklist. Data collectors were teams of 5 who were either national IMCI facilitator or supervisors. They spent 3 weeks to assess 24 facilities and this was because $25 \%$ of the facilities were reported either closed, cadre is not available or not functioning. The assessors observed IMCI performance of 24 health workers in clinical sessions with 24 children at Sharg-Alneel, as well as checking facilities' health system in term of availability of drugs and supplies relevant to the IMCI implementation. Once the assessment was completed, data was entered, cleaned and analyzed.

\subsection{Data Management and Analysis}

The questionnaires were checked for accuracy and completeness. When missing information was found, corrective measures were taken when possible. Data cleaning and entry was completed and analyzed using statistical package for the social science (windows version 21.0; SPSS). General descriptive analyses were used. Cross tabulations for variables that were thought to have an association were performed. The chi-square test was used as appropriate and $\mathrm{P}$-value of 0.05 was used to determine significance.

\section{Result}

A total of 500 caretakers were interviewed in the hospital survey. 497 were analyzed: 53.3\% from Alban -Jadeed, $32.4 \%$ from Om-Dawaban, 8\% from Abu-Dleg and 6.2\% from Wad-Abu-Salih hospitals.

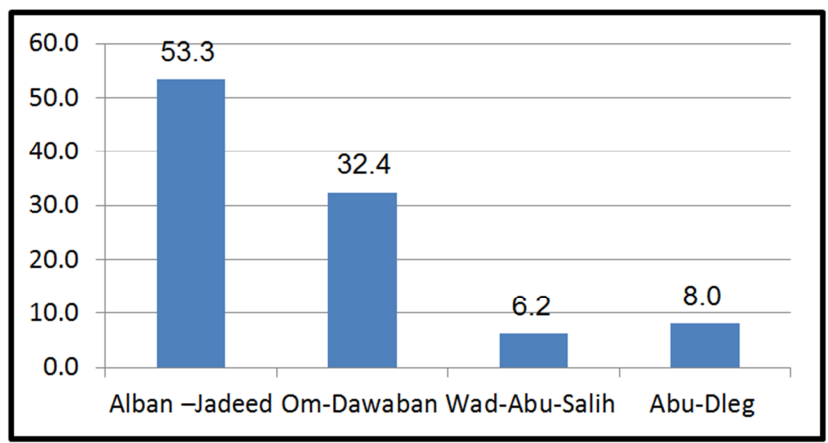

Figure 1. Percentage of caretakers interviewed by study hospital at ShargAlneel locality 2015. 
General description of the study participants

The proportion of female and male caretakers interviewed was $85.7 \%$ and $14.3 \%$ respectively. The mean age of the caretakers interviewed was 29.1 years and only $7 \%$ were not Sudanese. The majority of the female caretakers were the mothers $(84.9) \%$. $34 \%$ of the caretakers were illiterates, $23.7 \%$ had primary school education and only $4.4 \%$ had university education (table 1). 63\% of the caretakers interviewed stated that their total family spending was less than 15, 00 Sudanese pounds (SDG) per month (1 US $\$ \approx 12$ SDG).

The majority of children of the interviewed caretakers were boys $(326,65.4 \%)$ and the girls were 172 in number which is $34.6 \%$. The mean age of the children of caretakers interviewed was 17.4 months. Almost half $(242,49 \%)$ of the interviewed caretakers reported their children being sick within the past 1-2 days.

The most common symptoms reported were fever among 259 (52.1\%) children, diarrhea among 186 (37.4\%) children, and cough among 106 (21.3\%) children. These were followed by shortness of breathing among 109 (21.9\%), severe vomiting among $60(12.1 \%)$ children, not able to drink or breast feed among $24(4.8 \%)$ children, convulsion among $24(4.8 \%)$ children and stiff neck among $1(0.2 \%)$ child.

Children who had more than one of the main symptoms presented with fever and cough been $(40.69 \%)$, fever and diarrhea (24.5\%) and fever and vomiting (16.67\%).

Quantitative results (Bypass of PHC facilities)

$87 \%$ of interviewed care takers reported that they were self-referred (bypassing the public PHC Facilities) during the current child's sickness episode and only $12.9 \%$ were referred by health workers at other health facilities. About 304 caretakers interviewed (61\%) reported having a nearer facility other than the study hospital and $98 \%$ took less than 30 minutes walking distance to access such facilities. Of these, $79.6 \%$ had bypassed them during the current child's sickness episode.

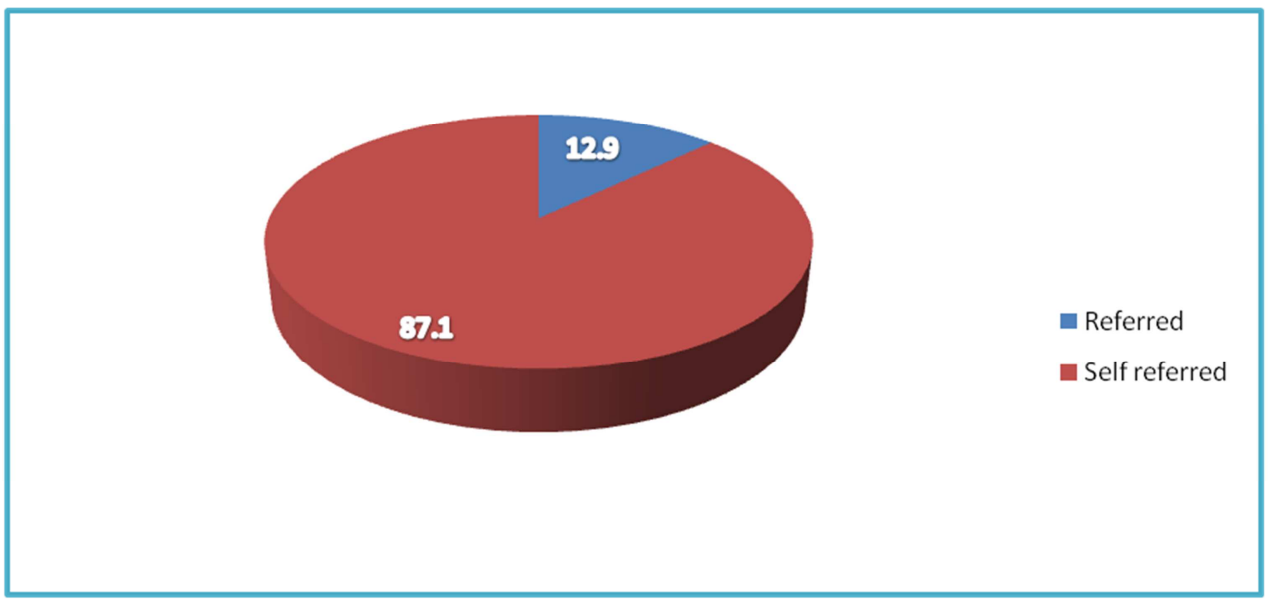

Figure 2. Proportion of caretakers seeking care for their under five children bypassing the PHC at Sharg-Alneel Locality, 2015.

Even though there are three Hospitals in Sharg-Alneel locality other than Alban-Jadeed, it is chosen by almost half of those bypassing PHC levels seeking care for common child illnesses. The main reason most frequently mentioned by the study participants for directly attending the chosen hospitals was that this hospital was the closest (43.9\%),followed by better health care quality at the hospital $(34.2 \%)$ and availability of doctors at the hospital (23.1\%). Few caretakers interviewed mentioned availability of drugs $(3.5 \%)$ and not knowing other health facilities available $(3.5 \%)$ as reasons for directly choosing the study hospital without referral.

Table 2. Bypass PHC facilities by general characteristics of the study participants.

\begin{tabular}{|c|c|c|c|c|}
\hline Variables & Frequency & \%age & $\%$ referral (non-bypassed) $(n=64)$ & $\%$ self-referral (bypassed) $(n=433)$ \\
\hline \multicolumn{5}{|c|}{ Caretakers sex $(n=497)$} \\
\hline Male & 71 & 14.3 & 14.06 & 14.3 \\
\hline Female & 426 & 85.7 & 85.9 & 85.7 \\
\hline \multicolumn{5}{|c|}{ Caretaker nationality $(\mathrm{n}=497)$} \\
\hline Sudanese & 463 & 93.2 & 93.75 & 93.07 \\
\hline Not Sudanese & 34 & 6.8 & 6.25 & 6.93 \\
\hline Mother & 422 & 84.9 & 84.375 & 84.99 \\
\hline Father & 43 & 8.6 & 10.9375 & 8.31 \\
\hline Other relatives & 32 & 6.4 & 4.6875 & 6.70 \\
\hline \multicolumn{5}{|c|}{ Caretakers' education $(n=497)$} \\
\hline Illiterate & 169 & 34 & 33 & 34 \\
\hline Primary & 162 & 32.6 & 31 & 33 \\
\hline
\end{tabular}




\begin{tabular}{|c|c|c|c|c|}
\hline Variables & Frequency & \%age & $\%$ referral (non-bypassed)(n=64) & $\%$ self-referral (bypassed) $(n=433)$ \\
\hline Intermediate & 28 & 5.6 & 3 & 6 \\
\hline Secondary & 112 & 22.5 & 25 & 22 \\
\hline University & 22 & 4.4 & 8 & 4 \\
\hline Postgraduates & 4 & 0.8 & 0 & 1 \\
\hline \multicolumn{5}{|l|}{ Caretakers' occupation $(\mathrm{n}=497)$} \\
\hline Housewife & 399 & 80.3 & 80 & 80 \\
\hline Free Business & 62 & 12.5 & 13 & 12 \\
\hline Private sector employee & 7 & 1.4 & 6 & 1 \\
\hline Public sector employee & 11 & 2.2 & 0 & 3 \\
\hline laborer & 12 & 2.4 & 0 & 3 \\
\hline Student & 5 & 1.0 & 2 & 1 \\
\hline Retried & 1 & 0.2 & 0 & 0 \\
\hline \multicolumn{5}{|c|}{ Average monthly spending $(n=497)$} \\
\hline$<1000$ (low) & 156 & 31.39 & 19 & 33 \\
\hline 1000- 2500 (medium) & 301 & 60.56 & 73 & 59 \\
\hline$>2500$ (high) & 40 & 8.08 & 8 & 8 \\
\hline \multicolumn{5}{|l|}{ Age of the child ( $n=497)$} \\
\hline 1 month- 12 & 234 & 47.1 & 66 & 44 \\
\hline $13-24$ & 150 & 30.2 & 25 & 31 \\
\hline $25-36$ & 61 & 12.3 & 9 & 13 \\
\hline $37-48$ & 35 & 7.0 & 0 & 8 \\
\hline 48- 60 & 17 & 3.4 & 0 & 4 \\
\hline \multicolumn{5}{|l|}{ Sex of the child $(n=497)$} \\
\hline Male & 325 & 65.4 & 78.13 & 63.5 \\
\hline Female & 172 & 34.6 & 21.88 & 36.49 \\
\hline \multicolumn{5}{|l|}{ Child illness symptoms $(n=497)$} \\
\hline Fever & 259 & 52.1 & 40.63 & 53.81 \\
\hline Diarrhea & 186 & 37.4 & 14.06 & 40.88 \\
\hline Cough & 106 & 21.3 & 15.63 & 22.17 \\
\hline SOB & 109 & 21.9 & 70.31 & 14.78 \\
\hline Vomit everything & 60 & 12.1 & 4.69 & 13.16 \\
\hline Convulsion & 24 & 4,8 & 7.81 & 4.39 \\
\hline Not able to drink or breast feed & 24 & 4.8 & 0 & 0.23 \\
\hline Stiff neck & 1 & 0.2 & 9.38 & 4.16 \\
\hline
\end{tabular}

Among those caretakers seeking care for their under-five children who were referred, only $30 \%$ contacted public PHC first at Sharg-Alneel locality. The majority of referred caretakers $(42 \%)$ contacted NGOs health facilities initially and the remainder (28\%) was referred from private clinics, pharmacy, local hospitals and traditional healers.

Bypassing public PHC facilities was found to be higher among caretakers of children who had fever (53.9\%), followed by diarrhea $(40.8 \%)$ and cough $(22.1 \%)$. The results also indicated that bypassing public PHC facilities was higher (79\%) among children with short duration symptoms of 1-4 days. The caretakers who bypassed the public PHC facilities with danger signs that coincided with the national IMCI guidelines were the majority $(73.3 \%)$.

The proportion of caretakers seeking care for their underfive children who bypassed the public PHC facilities for illnesses according to the national IMCI guidelines at ShargAlneel locality were presented as follows: 64 ill children had Shortness of Breathing (SOB) and 58 had vomiting of everything, while ill children with Convulsions, not able to drink or breastfed and neck stiffness were 19, 20 and only one respectively. Bypassing public PHC facilities was highest for the youngest age group (44.2\%) and it declined thereafter for older groups, where it reached 3.9\% for children older than 4 years. Bypassing public PHC facilities for common child illnesses for boys (64\%) was more than for girls $(36 \%)$.

More than half of the caretakers who bypassed public PHC facilities were between the ages of 20-30, while the lower percentage of bypassing was found to be among the age group of more than 50 years $(2 \%)$. Also the highest percentage of bypassing phenomenon was indicated among female $(86 \%)$ and married $(93 \%)$ caretakers.

With respect to the caretaker's nationality and relationship, the findings indicated that the proportion of caretakers who bypassed public PHC facilities were 93\% Sudanese and $85 \%$ biological mothers. Considering caretaker's education, results showed that bypassing PHC facilities for common child illnesses was higher (34 and 33\%) among illiterate and primary educated caretakers, while among the highly educated caretakers it was 5\%. Regarding caretakers occupation, respondents who were housewives reported the highest (80\%) self-referral proportion to hospitals.

Concerning caretaker's economic status, the influence of respondents' economic status on bypassing behavior was estimated using average monthly respondents' spending data. 
Respondents were divided into 3 groups (low, medium and high level spenders) according to their average monthly spending $(<1000$ SDG, 1000- 2500 SDG and $>2500$ SDG). Data analysis stated that bypassing public PHC facilities was elevated (92\%) among low and medium average monthly spending.

Association of bypassing (self-referral) with independent variables

To recognize the association between bypassing public PHC facilities for common child illnesses with the independent variables, chi-square test was used and then variables with $\mathrm{P}$-values less than 0.05 were considered being associated with the dependent variable.

Accordingly, the analysis revealed that bypassing behavior of public PHC services was statistically significantly different between child age groups $(\mathrm{P}=0.007)$ and child sex $(\mathrm{P}=0.02)$. Similarly, presenting symptoms of diarrhea $(\mathrm{P}=0.00004)$, fever $(\mathrm{P}=0.04)$, SOB $(\mathrm{P}=0.0003)$ and vomit everything $(\mathrm{P}=$ $0.05)$ were associated with bypassing public $\mathrm{PHC}$ facilities and self-referral to secondary health care facilities. The only identified caretaker's factors found to be associated with the bypassing behavior were occupation of the caretakers $(\mathrm{P}=$ $0.014)$ as well as the economic status $(\mathrm{P}=0.001)$.
Table 3. Association of bypassing (self-referral) with independent variables.

\begin{tabular}{ll}
\hline Independent variables & P value \\
\hline Child age & 0.007 \\
Child sex & 0.02 \\
Diarrhea & 0.00004 \\
Fever & 0.04 \\
Shortness of breathing & 0.0003 \\
Vomit every thing & 0.05 \\
Occupation & 0.014 \\
Economic status & 0.001 \\
\hline
\end{tabular}

The number of study participants who reported availability of closer health facilities was 303 (61.2\%), among those 161 (53.3\%) reported presence of public PHC facilities. 30.6\% of the study participants reported absence of nearest facility and $8.2 \%$ reported that availability of nearest facilities is unknown to them. Availability and knowledge about the existing PHC services was found to be statistically different between referred and self-referred respondents $(\mathrm{P}=0.001)$. Common reasons given why caretakers bypassing the closer public health facilities were unavailability of doctors $(\mathrm{P}=$ $0.0001)$, lack of health insurance services $(\mathrm{P}=0.001)$ and higher cost of services $(\mathrm{P}=0.001)$ (table 4$)$.

Table 4. Reasons for directly choosing the study hospitals mentioned by caretakers at Sharg-Alneel locality 2015.

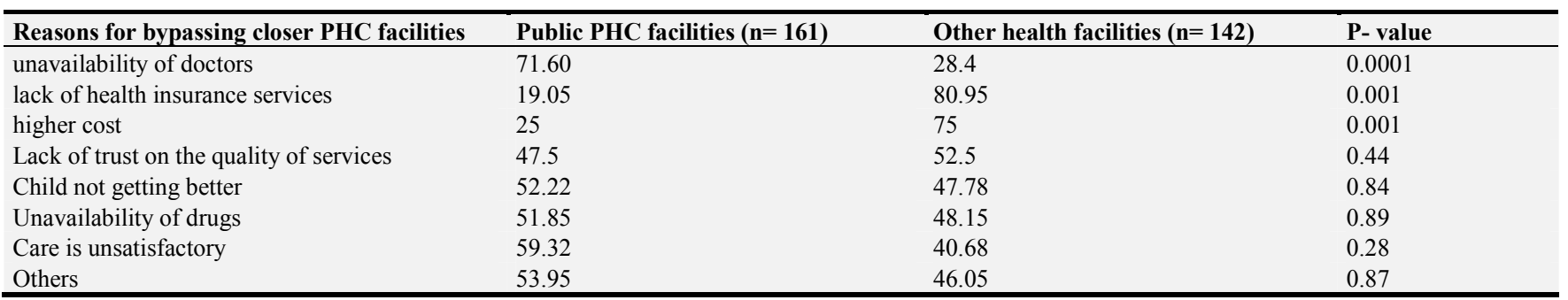

\section{Assessment findings}

Two third of health facilities assessed were Family Health centres. $70.8 \%$ of the health workers observed were males, $54.2 \%$ were doctors and $41.7 \%$ were medical assistants, and only $33 \%$ received IMCI training. Health care providers checked for child weight, temperature and current complain, percent being $83 \%, 50 \%$ and $96 \%$ respectively. $3 \%$ of health care providers checked child for all danger signs and 67 percent checked for child's four main symptoms.

Health care providers who checked for child's vaccination status, vitamin A supplementations and malnutrition status were $54.2 \%, 29.2 \%$ and $8.3 \%$ respectively. $25 \%$ of health care providers followed all the assessment steps in the IMCI guidelines accurately for cough cases followed by diarrhea $(12.5 \%)$, fever and ear problem (4.2\%). Less than $5 \%$ of health care providers were correctly "classified" (IMCI terminology meaning diagnosed) cases of cough and ear problem. No health workers classified cases presenting with fever and diarrhea correctly. Among 16 children who required an emergency transfer during the assessment, 12 were referred. Almost $83 \%$ of children who were referred $(12 / 16)$ received a pre-referral treatment. In addition, only $50 \%$ of health care providers treated cases classified as having ear problem correctly, while $90 \%$ and $70 \%$ correctly treated classified children having malaria and diarrhea correspondingly. Findings concerning communication and counseling revealed that percentage of health care providers who advised caretakers on rules of home treatment and nutrition/feeding was $41.7 \%$, while those who advised on when to immediately reconsider the PHC were only $12.5 \%$.

$65 \%, 66.7 \%$ and $65.8 \%$ of health care providers checking for child weight, temperature and current complain respectively were not trained on IMCI. All health care providers who checked children for danger signs received IMCI training. More than $50 \%$ of health care providers who checked for child's four main symptoms did not receive IMCI training. Health care providers who received IMCI training and checked for child's vaccination status, vitamin A supplementations and malnutrition status were $46.2 \%, 42.9 \%$ and $100 \%$ respectively. All health care providers followed all the assessment steps in the IMCI guidelines accurately for diarrhea, fever and ear problem cases and were trained on IMCI. All health care providers who correctly "classified" (IMCI terminology meaning diagnosed) cases of cough and ear problem received IMCI training while only half of those who classified cases of malnutrition were correctly trained. Only one third of HCP who transfer children requiring an emergency referral were trained on IMCI.HCP who received 
IMCI training andprescribed pre-referral treatment at the PHC (anti-malarial and/or antibiotics) were 30\%. In addition, $100 \%$ of health care providers treated cases classified as having ear problem correctly and they did receive IMCI training, while $71.4 \%, 44.4 \%$ and $28.6 \%$ correctly treated classified children having pneumonia, malaria and diarrhoea correspondingly and were trained on IMCI. More than half of health care providers who advised caretakers on rules of home treatment and nutrition/feeding did not receive IMCI training, while two third of HCP who advised on when to immediately transfer to the PHC received IMCI training.
Thirty six indicators related to the health system support for the management of cases through IMCI in health centers in sharg-Alneel locality have been calculated. The analysis was limited to the availability on the day of the visit. They were divided into three categories: the availability of essential equipment, availability of essential IMCI drugs and availability of pre-referral drugs. The assessment reported that no health facilities had all items of equipment, material and essential IMCI drugs. Percentage of health facilities which had all items of the pre- referral drugs was $4.2 \% .37 \%$ of health facility had free drugs for under-5children.

Table 5. Health workers performance by IMCI training regarding assessment, classification, management and counseling of under-5 sick child at health facilities in Sharg-Alneel locality.

\begin{tabular}{|c|c|c|}
\hline Indicators & \% Received IMCI training & $\%$ Not received IMCI training \\
\hline \multicolumn{3}{|l|}{ Assessment of case } \\
\hline HCP who checked child weight & 35 & 65 \\
\hline HCP who checked child temperature & 33.3 & 66.7 \\
\hline HCP who checked child current complain & 34.2 & 65.8 \\
\hline HCP who checked child for danger sign & 100 & 0 \\
\hline HCP who assessed main symptoms (cough) & 46.7 & 53.3 \\
\hline HCP who assessed main symptoms (diarrhoea) & 43.8 & 56.2 \\
\hline HCP who assessed main symptoms (Ear problems) & 83.3 & 16.7 \\
\hline HCP who checked child with malnutrition correctly & 100 & 0 \\
\hline HCP who checked for child's vaccination status & 46.2 & 53.8 \\
\hline HCP who checked for vitamin A supplementations & 42.9 & 57.1 \\
\hline HCP who assessed child with cough correctly & 66.7 & 33.3 \\
\hline HCP who assessed child with fever correctly & 100 & 0 \\
\hline HCP who assessed child with diarrhea correctly & 100 & 0 \\
\hline HCP who classify child with cough correctly & 100 & 0 \\
\hline HCP who classify child with fever correctly & 0 & 0 \\
\hline HCP who classify child with fever for measles correctly & 0 & 0 \\
\hline $\begin{array}{l}\text { HCP who classify child with diarrhoea for dehydration, persistency and dysentery } \\
\text { correctly }\end{array}$ & 0 & 0 \\
\hline HCP who classify child with ear problem correctly & 100 & 0 \\
\hline HCP who classify child with malnutrition correctly & 50 & 50 \\
\hline \multicolumn{3}{|l|}{ Management of cases } \\
\hline HCP who transfer of children requiring anemergency reference & 33.3 & 66.7 \\
\hline $\begin{array}{l}\text { HCP who prescribed and gave Pre-referral treatment at the PHC (anti-malarial } \\
\text { and/or antibiotics) }\end{array}$ & 30 & 70 \\
\hline HCP who did noted referral & 20 & 80 \\
\hline HCP who treated Pneumonia properly & 71.4 & 28.6 \\
\hline HCP who treated Malaria correctly & 44.4 & 55.6 \\
\hline HCP who treated Diarrhea properly (- ORS only) & 28.6 & 71.4 \\
\hline \multicolumn{3}{|l|}{ Counselling and communication } \\
\hline HCP who advised to return for follow up & 50 & 50 \\
\hline $\begin{array}{l}\mathrm{HCP} \text { who advisedon rules of home treatment: give moreliquid and continue } \\
\text { feeding duringillness }\end{array}$ & 45.5 & 54.5 \\
\hline $\mathrm{HCP}$ who advisedwhen to return immediately to the PHC & 66.7 & 33.3 \\
\hline
\end{tabular}

\section{Discussion}

Main objective of Sudan PHC reform 2012-2016 was to increase population access to PHC services from $86 \%$ to $100 \%$ with the availability of free health care services policy for under-5 children. However, the real use of the service has been low, extremely restricting the overall effectiveness of the health system response. This study identified the magnitude of and factors influencing bypassing PHC services for common child hood illnesses in Sudan. This chapter discusses the findings from the study in section 3. It is organized on the major elements of the study objectives and included the findings from other low and middle income countries.

The result revealed that the majority of caretakers were uneducated young mothers. This might be due to the interview was conducted during the official working hours of 
the head of the family. The majority of children were boys presenting with fever of short duration.

The findings showed that though $45.3 \%$ of caretakers interviewed took from more than 30 minutes to more than 180 minutes to reach the study hospital, $87 \%$ of caretakers interviewed had sought care for their under-5 children directly from secondary hospital without referral. This is in line with other findings from other low income countries such as Uganda, Nigeria, Tanzania, and Namibia [14, 15, and 16]. The main common reasons given for directly choosing the study hospitals were the hospitals are nearer than PHC services, better quality of health care services and availability of doctors at secondary level. Of the total interviewed Caretakers who bypassed, 61\% have nearer PHC services which they could have utilized, among these $98 \%$ were less than 30 minutes walking distance. $43.9 \%$ of bypassed caretakers reported that hospital is closer, among those $30.5 \%$ reported that they did not have nearer PHC facilities and $8.4 \%$ did not know about the availability of the closer PHC services. Therefore, $5.1 \%$ reported that they had physical access to PHC facilities but they perceived that the hospital is closer.

The main common reason given for bypassing nearer PHC facilities was unavailability of doctors which was consistent with national characterization of health facilities. PHC services staffed by medical doctors were FHC and local hospitals located at urban areas while rural areas were only staffed by paramedics [17]. However, Sudan has started to implement in-services family medicine project in Gezira state which succeeded in recruiting 207 medical doctors providing PHC services in 158 Family Health Centers, of which more than half had never been served by a doctor before [18].

The second most common reason given for bypassing PHC facilities was lack of health insurance services. The FMoH policy emphasizes the important position of (PHC). Inversely, the National Health Insurance Fund's (NHIF) investment is mainly on tertiary and secondary care. NHIF in Sudan purchase services from governmental Family Health centre only at the Primary Health services level [19]. According to Health Insurance Khartoum State (HIKS) report 2015, service coverage is $67 \%$ of the governmental Family Health centre all over Khartoum state. Though NGOs services are not included in health insurance services coverage, this study reported that they are widely available and more utilized than governmental one in the study area. Low and non-inclusive health insurance service coverage could justify our study findings.

Free care for pregnant women and under-fives was announced by the President, January 2008. Nevertheless, another reason for bypassing PHC facilities given by caretakers in our study was high cost of services. This is consistent with our qualitative findings and facility assessment finding which found that only $37 \%$ of PHC facilities have free drugs for under- 5 children. Findings from other policy evaluation study that showed Sudan free care policy are highly needed but are poorly specified, funded, implemented and monitored [20]Obviously this result in low participation of health facilities in policy implementation and hence continuation of fee for services and drug sales.

Caretakers and child factors were identified by the study to influence bypassing PHC services for common child illnesses. Our study found that child gender affects the decision to choose a health care provider. Bypassing public PHC services for common childhood illness was more for boys than girls and this could be attributed to the society pediatrics' gender bias, explained by existence of strong sonpreference in Sudan, in line with studies of gender bias and health seeking actions in other African courtiers including Egypt, Tunisia, Morocco, and Nigeria [21-23]. Same findings are evident also from several studies from Asia including Nepal, Pakistan and China and Republic of Korea [24-26]. All the studies suggested that gender role affects the entire steps of child health care seeking action. This study suggests that proportion of caretakers bypassing PHC statistically significantly differ by child age group which was noted to be higher among younger age. This observation was also reported by many other countries. Whereas a study conducted in Tanzania showed no significant difference between the caretakers bypassing their nearest facility according to child's age [15].

Among caretaker's socio-demographic characteristics, only occupation and economic status of the respondents was significantly associated with self-referral (bypassing). These findings are in accordance with other studies from Kenya, Papua New Guinea, China and Nepal [27-31]. Even though, the association was not statistically significant, other caretakers socio-demographic factors also positively affected bypassing behavior especially age and education. Several studies have reported positive association between caretakers' education and bypassing PHC services. Surprisingly, this study suggested that bypassing behavior was not significantly different across education status groups and uneducated bypassed the primary levels of healthcare more than highly educated caretakers. These findings can be explained by some reasons including the fact that respondents have no trust in the care they would receive at PHC level and lack of adequately designed and performing referral system.

Bypassing behavior was strongly related to particular illness symptoms and their perceived severity. Our study indicated that presenting symptoms with diarrhea ( $\mathrm{P}$ $=0.00004)$ and fever $(\mathrm{P}=0.04)$ were significantly associated with bypassing PHC services. This might be due to caretaker's sensitivity towards diarrheal and fever symptoms than coughing. Our research findings suggested that children suffering from severe illness such as SOB and vomit everything were associated with bypassing public PHC facilities and self-referral to secondary health care facilities. This could be because caretakers are expected to seek higher quality health services for severe illnesses. It might also be due to the previous experience of caretakers with inadequately performing referral services. These findings were also reported in other countries [29, 30, and 7].

Results of assessment of the selected public PHC facilities 
showed that $25 \%$ of the facilities were reported either closed, cadre is not available or not functioning. Closed was indicated by either short opening hours or closed on the day of the visit. Assessors also found that $6.25 \%$ were open but medical cadres were not available. Moreover, local community reported to assessment team that $12.5 \%$ of the facilities were not functioning for long time ago.

Findings on the performance of health workers revealed that all the four components of the case management were still weak particularly systematic assessment and classification. These results were comparable to those obtained during other assessments such as in Rwanda and Kenya [32, 33]. Poor compliance of health workers to IMCI guidelines was obviously noted. For example, health providers checked for all danger signs in only $12.5 \%$ of the children observed, and they did not check for all major symptoms in the children. Less than quarter of the health care providers followed all the assessment steps in the national IMCI guidelines accurately. Less than one-third were correctly classified. However, percentage of health care providers who treated sick children correctly approached $90 \%$ based on the disease. Communication and counseling are crucial for proper continuous care at home as well as appropriate family behavior. Nevertheless, this study showed that only $40 \%$ of the health care providers communicated with caretakers appropriately and provided counseling regarding important issues. Gaps reported in the performance of health workers could be clarified by lack of training as only one third of the observed health workers were trained on IMCI guidelines. Moreover, quality of training was also an issue, as 8 out of 15 and 6 out of 8 procedures of case assessment and case management respectively were performed more properly among HCP who did not receive IMCI training. Other explanation could be poor monitoring and supervision, in addition to the resistance from the health care providers themselves to apply IMCI because it is time consuming and a burden to their workload [32].

The support system of the study primary health facilities were not adequately functioning. This study showed that only $12.5 \%$ of assessed primary health facilities had $95 \%$ of the essential equipment and material. Similarly, $12.5 \%$ of health facilities had $78 \%$ of the essential IMCI drugs. No health facility reported having all items of the essential equipment and IMCI drugs. All items of Pre-referral drugs were available in only one primary health facility $(4 \%)$. These findings were consistent with other studies conducted in Morocco and Tanzania [34] but not with study from Rwanda [32].

Findings of the two study methods were consistent and supporting each other. All determinants of bypassing behavior reported were mainly quality dimensions, availability, accessibility, adequacy, acceptability and affordability of services. Both methods showed that either PHC services were not available or study respondents were not aware about the existing services or they perceived that the hospital was nearer. If this indicates anything, it showed inequitable distribution of health services and discrepancy between services distribution and referral policy. Also the two study approaches were reporting consistent findings with regard to the poor quality of PHC services indicating weak PHC services monitoring and control.

\section{Conclusion}

This is one of the first researches to study magnitude of and factors influencing the bypassing PHC services for common childhood illnesses in Sudan. This study has shed light on the magnitude of the problem and different individual, illness, provider and system factors that prevent caretakers from using closer PHC services and directly choose secondary hospital.

This study indicated that almost 9 of 10 care takers interviewed had directly chosen the secondary level without referral despite extra time and cost. Among those who were referred, for only $30 \%$ the source of referral was public PHC facilities. The main reason given for directly choosing the hospitals was better quality of care.

Child factors including age and sex were both found to be significantly associated with bypassing public PHC services for common child illness.

This study suggested that bypassing PHC services for common child illness was not statistically different between caretaker age, nationality, relationship, and education group, unlike occupation and economic status that were found to be significantly associated with bypassing behaviour. Lack of knowledge about existing PHC services among the study participants and perception of hospital's closeness also emerged to influencing choice of PHC services.

The main reasons given for bypassing the nearer PHC services were unavailability of doctors, health insurance services as well as high cost of services.

Institutional assessment reported that quarter of selected health facilities were either not adequately functioning or not functioning at all. Still there is gap in the performance of health workers in implementing IMCI guidelines in addition to health system supporting IMCI implementation

\section{Recommendations}

In order to reduce the bypassing phenomenon in Sudan we recommend Federal and State Ministry of Health, in collaboration with all relevant stakeholders, to implement the following recommendations based on the findings of this study.

Intervention recommendation

- Ensure availability of doctors (expand the implementation of family medicine policy, revisit staffing of PHC)

- Scale up of health insurance coverage including NGOs services

- Enhance implementation of free care policy

- Improve the working setting and strengthening health system at the PHC facilities

- Scale up coverage and quality of IMCI training and 
strengthening supervision in the PHC services where IMCI is implemented to improve the performance of health workers

Enabling environment recommendations

- Develop an overarching Policy that ensures quality, equity and gate keeping

- Promote Community mobilization (Focus IEC programme)

- Improve and support implementation of referral system

Monitoring and evaluation and research recommendation:

- Strengthening of monitoring and supervision to ensure the quality and availability of the PHC services,

- Conduct more analytical studies on health-seeking behaviour and utilization of PHC services.

\section{References}

[1] FMOH: Promoting access to high quality primary health care services in Sudan (policy brief). Khartoum: Policy and Planning Unit 2011.

[2] FMOH: Sudan Household Health survey-Round 2. Khartoum: Policy and Planning Unit 2012.

[3] FMOH: National Acceleration PLAN for Maternal and CHILD Health 2013 -2015. Khartoum: Directorate General for PHC and Preventive Medicine 2013.

[4] WHO: Reduction of child mortality in the Eastern Mediterranean Region. Geneva: World Health Organization 2012.

[5] Macinko J, Starfield B, Erinosho T. The impact of primary healthcare on population health in low- and middle-income countries. J Ambul Care Manage. 2009; 32(2):150.

[6] Darmstadt GL, Bhutta ZA, Cousens S, Adam T, Walker N, de Bernis L: Evidence-based, cost-effectiveinterventions: how many newborn babies can we save? Lancet 2005, 365(9463):977-88.

[7] Rutherford ME, Mulholland K, Hill PC: How access to health care relates to under-five mortality in sub-Saharan Africa: systematic review. Trop Med Int Health 2010, 15(5):508-19.

[8] Low A, Coeyere D, Shivute N, Brandt LJ: Patient referral patterns in Namibia: identification of potential to improve the efficiency of the health care system. Int J Health Plan M 2001, 16(3):243-57.

[9] Audo MO, Ferguson A, Njoroge PK: Quality of health care and its effects in the utilization of maternal and child health services in Kenya. East Afr Med J 2006, 82(11).

[10] Sembuche SH, Ishengoma DS, Seth MD, Francis F, Rutta AS, Kamugisha ML, Lemnge MM: Epidemiology of malaria in an area prepared for clinical trials in Korogwe, north-eastern Tanzania. Malaria Journal 2009, 8:165.

[11] Kruk ME, Mbaruku G, McCord CW, Moran M, Rockers PC, Galea S: Bypassing PHC facilities for childbirth: a population-based study in rural Tanzania.Health Policy Plan 2009, 24(4):279-88.

[12] FMOH: Knowledge, Attitudes and Practices (KAP) in the Sudanese communities for their health seeking behavior:
Policy and Planning Unit 2011.

[13] KSMOH: Annual statistical report 2013: health information management system directorate 2013 (unpublished).

[14] Rutebemberwa E, Kallander K, Tomson G, Peterson S, Pariyo G: Determinants of delay in care seeking for febrile children in eastern Uganda. Trop Med \&Int Health 2009, 14(4):472-9.

[15] Kahabuka et al.: Why caretakers bypass Primary Health Care facilities for child care - a case from rural Tanzania. BMC Health Services Research 2011, 11:315.

[16] Low A, Coeyere D, Shivute N, Brandt LJ: Patient referral patterns in Namibia: identification of potential to improve the efficiency of the health care system. Int J Health Plan M 2001, 16(3):243-57.

[17] FMOH: Specifications and standards for the health system in Sudan, Part II: Document of Characterization of services and health units. Khartoum: Policy and Planning Unit 2010.

[18] Mohamed et al: Scaling up family medicine training in Gezira, Sudan - a 2-year in-service master programme using modern information and communication technology: a survey study, Human Resources for Health 2014, 12:3.

[19] FMOH, Health Financing System Review Report, May 2014, Public Health Institute, unpublished.

[20] Free healthcare policy for under-fives and pregnant women in northern Sudan:findings of a review. Khartoum: directorate general of planning and international health 2011.

[21] Yount KM: Gender bias in the allocation of curative health care in Minia, Egypt. Popul Res Policy Rev 2003; 22:267-95.

[22] Pauline Rossi, Lea Rouanet. Gender Preferences in Africa: A Comparative Analysis of Fertility Choices. PSE Working Papers n 2014-33. 2015. <halshs-01074934v2>.

[23] Milazzo, A. M. Son preference, fertility and family structure. Evidence form reproductive behavior among Nigerian women. World Bank Policy Research Working Paper 2014, No. 6869 .

[24] S. Pokhrel et al: Gender role and child health care utilization in Nepal. Health Policy 2005;74100-109.

[25] Nuruddin R, Hadden WC, PetersenMR, Lim MK: Does child gender determine household decision for health care in rural Thatta, Pakistan? J Public Health (Oxf) 2009; 31:389-97.

[26] Das Gupta, Metal. "Why is Son Preference so Persistent in East and South Asia? A Cross Country Study of China, India and the Republic of Korea." Journal of Developmental Studies, (2003) vol.40 (2).

[27] Audo MO, Ferguson A, Njoroge PK: Quality of health care and its effects in the utilization of maternal and child health services in Kenya. East Afr Med J 2006, 82(11).

[28] Tsukahara T., The Choice of Healthcare Providers for Febrile Children after Introducing Non-professional Health Workers in a Malaria Endemic Area in Papua New Guinea. 2015; $3: 275$.

[29] Dong et al: Focus on vulnerable populations and promoting equity in health service utilization - an analysis of visitor characteristics and service utilization of the Chinese community health service. BMC Public Health 2014, 14:503. 
[30] Wu D, Lam TP. Underuse of Primary Care in China: The Scale, Causes, and Solutions. JABFM 2016, Vol. 29 No.

[31] Sreeramareddy C T, et al: Care seeking behavior for childhood illness- a questionnaire survey in western Nepal. BMC International Health and Human Rights 2006, 6:7.

[32] $\mathrm{MOH}$, Report of IMCI evaluation in the District of Kirehe in Rwanda - July 2008. Rwanda.
[33] Lin Y. and Tavrow P: Assessing Health Worker Performance of IMCI in Kenya. Quality Assurance Project Case Study. Published for the U. S. Agency for International Development (USAID) by the Quality Assurance Project (QAP): 2000 Bethesda, Maryland, U.S.A.

[34] Tulloch J: Integrated approach to child health in developing countries. The Lancet 354 (Suppl. 2): SII16-20. 